FERMILAB-Conf-95/281-T

\title{
Potential for Discoveries in Charm Meson Physics t
}

\author{
Gustavo Burdman \\ Fermi National Accelerator Laboratory, P. O. Box 500, Batavia, IL 60510, USA.
}

\begin{abstract}
The possibility of using charm meson physics to test the Standard Model (SM) is reviewed. In the case of $D^{0}-\bar{D}^{0}$ mixing, the SM contributions are carefully considered and the existence of a window for the observation of new physics is discussed. Some examples of extensions of the SM giving large mixing signals are presented. Finally, some distinctive aspects of $\mathrm{CP}$ violation and rare decays in charm mesons are discussed.
\end{abstract}

The $D$ meson has been largely overlooked as a testing ground for the SM. The reason for this might be traced back to the fact that the most important effects in Flavor Changing Neutral Currents (FCNC) and in flavor mixing are brought about, in the SM, by the top quark. This has important effects in loops that couple to external down quarks ( $B$ mixing, radiative and rare $B$ and $K$ decays). In the SM, top quark loops do not couple to external up quarks and thus the SM loop effects in charm physics are expected to be very small. This is the case, for instance, for $D^{0}-\bar{D}^{0}$ mixing and for rare decays: the SM predicts very small rates. However this can be viewed as a window of opportunity for observing effects coming from new physics at higher energy scales. Of these, the most interesting ones are those that are relatively small or even negligible in $B$ and $K$ physics but become observable contributions when looking at $D$ physics. Here we will discuss where these opportunities are likely to be and some of the new physics scenarios giving interesting signals. We will

\footnotetext{
${ }^{1}$ Talk delivered at the Workshop on the Tau/Charm Factory, Argonne June 21-23 1995.
} 
first discuss $D^{0}-\bar{D}^{0}$ mixing in the SM, focusing on our current understanding of the long distance contributions. This is a crucial point in order to establish whether or not there is a window to observe new physics in the measurement of this effect. Then we will go on to show some examples of extensions of the SM that could fill this experimental window. Among these are multi-Higgs doublet models, fourth-generation effects, supersymmetry, and tree-level FCNC effects induced by dynamical symmetry breaking scenarios. We will then move to briefly review the prospects of CP violation effects in $D$ physics, both direct and associated with mixing, in the SM and beyond. Finally, we will take a look at radiative and rare decays and point out the relevance of some modes as tests of the SM.

\section{$D^{0}-\bar{D}^{0}$ MIXING IN THE STANDARD MODEL}

The current experimental knowledge of $D^{0}-\bar{D}^{0}$ mixing comes from the upper bound on the wrong-sign to right-sign ratio

$$
r_{D} \equiv \frac{\Gamma\left(D^{0} \rightarrow \ell^{-} X\right)}{\Gamma\left(D^{0} \rightarrow \ell^{+} X\right)} \simeq \frac{1}{2}\left[\left(\frac{\Delta m_{D}}{\Gamma}\right)^{2}+\left(\frac{\Delta \Gamma_{D}}{\Gamma}\right)^{2}\right],
$$

with the approximation in (11) valid for $\Delta m_{D} / \Gamma, \Delta \Gamma_{D} / \Gamma \ll 1$. From the latest E691 data [1] we know $r_{D}<3.7 \times 10^{-3}$. If $\Delta \Gamma_{D} / \Gamma$ is neglected, this translates into an upper limit for the mass difference giving

$$
\Delta m_{D}^{\text {exp. }}<1.3 \times 10^{-13} \mathrm{GeV}
$$

\section{Short Distance}

In the $\mathrm{SM}$ the short distance $\Delta C=2$ transition occurs via the box diagrams. The effective interactions at the $m_{c}$ scale are described by the hamiltonian [2]:

$$
\mathcal{H}_{\text {eff. }}^{\Delta C=2}=\frac{G_{F}}{\sqrt{2}} \frac{\alpha\left|V_{c s}^{*} V_{u s}\right|^{2}}{8 \pi \sin ^{2} \theta_{W}} \frac{\left(m_{s}^{2}-m_{d}^{2}\right)^{2}}{m_{W}^{2} m_{c}^{2}}\left(\mathcal{O}+\mathcal{O}^{\prime}\right)
$$

with $\mathcal{O} \equiv \bar{u} \gamma_{\mu}\left(1-\gamma_{5}\right) c \bar{u} \gamma^{\mu}\left(1-\gamma_{5}\right) c$ and $\mathcal{O}^{\prime} \equiv \bar{u}\left(1+\gamma_{5}\right) c \bar{u}\left(1+\gamma_{5}\right) c$. The presence of the additional operator $\mathcal{O}^{\prime}$ is due to the non negligible external momentum. The matrix elements of the operators can be parametrized by

$$
\left\langle D^{0}|\mathcal{O}| \bar{D}^{0}\right\rangle=\frac{8}{3} m_{D} f_{D}^{2} B_{D} \quad ; \quad\left\langle D^{0}\left|\mathcal{O}^{\prime}\right| \bar{D}^{0}\right\rangle=-\frac{5}{3}\left(\frac{m_{D}}{m_{c}}\right)^{2} m_{D} f_{D}^{2} B_{D}^{\prime}
$$

In the vacuum insertion approximation one has $B_{D}=B_{D}^{\prime}=1$ and the short distance contribution to the mass difference is

$$
\Delta m_{D}^{\mathrm{SD}} \simeq 2.5 \times 10^{-17} \mathrm{GeV}\left(\frac{m_{s}}{0.3 \mathrm{GeV}}\right)^{4}\left(\frac{f_{D}}{f_{\pi}}\right)^{2}
$$


Thus for typical values of $f_{D}$ and $m_{s}$ the short distance contributes to $r_{D}$ with a value not above $10^{-8}$, perhaps as small as $10^{-10}$.

\section{Long Distance}

The contributions of the short distance box diagrams are not the only ones. The fact that light quarks with rather large CKM couplings to the charm quark can propagate between the $D^{0}$ and $\bar{D}^{0}$, hints the possibility of relatively important long distance contributions to mixing. The propagating degrees of freedom are hadrons rather than quarks. The situation is very different in $K$ and $B$ mixing, where there is always a very important effect of a heavy quark inside the box diagram loop, with large CKM couplings: the charm quark in $K^{0}-\bar{K}^{0}$ and the top quark in $B^{0}-\bar{B}^{0}$. In the latter, the effect of the top quark completely dominates and long distance contributions are expected to be negligibly small due to the small CKM couplings of the $B$ meson to light hadrons. In the case of $K$ mixing, the coupling to light-hadron intermediate states is still large as a consequence of which sizeable long distance contributions - of the same order of magnitude of the short distance ones- are expected [3]. The long distance contributions to $D^{0}-\bar{D}^{0}$ mixing are inherently nonperturbative and cannot be calculated from first principles. It is however of paramount importance to estimate their size in order to understand to origin of a possible observation of the effect in future experiments.

A first observation is that the mass difference is a $S U(3)$ breaking effect. On the other hand, $\Delta m_{D}$ is doubly Cabibbo suppressed whereas $\Gamma$ is not. Therefore, a naive estimate of the effect would be given by

$$
\frac{\Delta m_{D}}{\Gamma} \sim \lambda^{2} \times(S U(3) \text { breaking }) \simeq\left(10^{-3}-10^{-2}\right)
$$

where $\lambda \simeq \sin \theta_{c}$. Specific calculations tend to give smaller results. There are basically two ways to attempt estimating the long distance effects: a dispersive approach and Heavy Quark Effective Theory (HQET).

\section{Dispersive Approach}

An estimate of the long distance contributions can be obtained by assuming they come from the propagation of hadronic states to which both $D^{0}$ and $\bar{D}^{0}$ can decay. There will be one, two, three, etc. particle intermediate states. Each of these groups can be further separated into sets whose contributions vanish separately in the $S U(3)$ limit. One of these sets is formed by the twocharged-pseudoscalar intermediate states $\pi^{+} \pi^{-}, K^{+} K^{-}, K^{-} \pi^{+}$and $K^{+} \pi^{-}$. Thus computing their contribution to the mass difference, as shown schematically in Fig. 1, gives a concrete realization of the estimate in (6) for an $S U(3)$ set for which data is available. This was first done in [4]. 


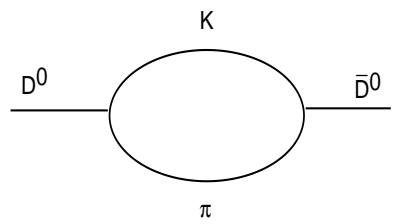

Figure 1: Long distance contribution from two charged pseudo-scalar intermediate states.

Although these "self energy" diagrams will depend on the interaction chosen for the vertices, they have a universal imaginary part which typically comes from a logarithm. This leads to the expression [4]

$$
\frac{\Delta m_{D}^{l . d .}}{\Gamma} \simeq \frac{1}{2 \pi} \ln \frac{m_{D}^{2}}{\mu^{2}}\left[B\left(\pi^{+} \pi^{-}\right)+B\left(K^{+} K^{-}\right)-2 \sqrt{B\left(K^{-} \pi^{+}\right) B\left(K^{+} \pi^{-}\right)}\right]
$$

where all the branching ratios correspond to $D^{0}$ decays. The scale $\mu$ is a typical hadronic scale, $\mathcal{O}(1 \mathrm{GeV})$. Inserting the current available experimental values [5] in (7) we obtain

$$
\frac{\Delta m_{D}}{\Gamma} \simeq 8.4 \times 10^{-4} \times(1.46-\sqrt{b})
$$

where we have defined $b$ by

$$
\frac{B\left(K^{+} \pi^{-}\right)}{B\left(K^{-} \pi^{+}\right)}=b \tan ^{4} \theta_{c}
$$

The experimental measurement of the Doubly Cabibbo Suppressed Decays (DCSD) gives $b=2.8 \pm 1.3$. Then, for any value of $b$ in a $1 \sigma$ interval, it is clear that the cancellation in this $S U(3)$ set is even better than expected in (6), giving $\left|\Delta m_{D}^{\text {l.d. }} / \Gamma\right| \simeq \mathcal{O}\left(10^{-4}\right)$.

Several assumptions are implied in (7). First, the minus sign is obtained assuming there is no strong relative phase between the two $K \pi$ amplitudes. This is only true in the $S U(3)$ limit [6]. However, the effect of this phase is expected to be small [7]. More importantly, equation (7) is valid for massless particles in the loop and it assumes a constant coupling at the vertices which allows one to relate the product of the two $\Delta C=1$ interactions in Fig. 1 to the actual decay amplitudes. The approximation regarding the internal masses is rather safe in the two-pseudoscalar (PP) case. However masses should be kept in the calculation when sets including vector mesons (PV and VV) are considered. On the other hand, large momenta in the loop should not contribute given that the coupling is expected to develop a momentum suppression at a 
typical hadronic scale. This suggests the existence of a physical cutoff for the integrals involved. It is instructive to see how this cutoff and the effect of the internal masses come about. The contribution of Fig. \& to the mass difference obeys a dispersion relation of the form

$$
\Sigma\left(p^{2}\right)=\frac{1}{\pi} \int_{s_{0}}^{\infty} \frac{\operatorname{Im}[\Sigma(s)] d s}{\left(s-p^{2}-i \epsilon\right)}
$$

where $s_{0} \equiv\left(m_{1}+m_{2}\right)^{2}$, and $m_{1}$ and $m_{2}$ are the masses in the loop. Taking into account a subtraction forcing the condition $\Sigma(0)=0$ and keeping the masses, the implementation of a cutoff $\Lambda$ in the dispersive integral (10) gives [9]

$$
\begin{aligned}
\frac{\Delta m_{D}^{\text {l.d. }}}{\Gamma} \simeq \frac{m_{D}}{4 \pi} & \left\{\frac{B\left(\pi^{+} \pi^{-}\right)}{\mathbf{p}_{\pi \pi}} I\left(m_{\pi}, m_{\pi}, \Lambda\right)+\frac{B\left(K^{+} K^{-}\right)}{\mathbf{p}_{K K}} I\left(m_{K}, m_{K}, \Lambda\right)\right. \\
& \left.-2 \frac{\sqrt{B\left(K^{-} \pi^{+}\right) B\left(K^{+} \pi^{-}\right)}}{\mathbf{p}_{K \pi}} I\left(m_{\pi}, m_{K}, \Lambda\right)\right\}
\end{aligned}
$$

where $\mathbf{p}_{i j}$ is the magnitude of the three-momentum in the actual decay and

$$
I\left(m_{1}, m_{2}, \Lambda\right)=-\int_{s_{0}}^{\Lambda^{2}} \frac{\sqrt{1-\frac{s_{0}}{s}} d s}{s-m_{D}^{2}}
$$

If the massless limit is taken in (11) one recovers (7) with the identification $\mu^{2}=2 m_{D}\left(\Lambda-m_{D}\right)$. Although the result depends strongly on the cutoff $\Lambda$, this can be interpreted as the value of $s$ for which the internal momentum reaches its maximum. Not surprisingly, the value of $\Lambda$ giving an internal momentum of $\sim 1 \mathrm{GeV}$ is the same giving $\mu \sim 1 \mathrm{GeV}$. This is $\Lambda \simeq(2-2.2) \mathrm{GeV}$, not too far above $m_{D}$. Using this cutoff results in a contribution to the mass difference of

$$
\left.\frac{\Delta m_{D}^{\text {l.d. }}}{\Gamma} \simeq 6.5 \times 10^{-4} \times(1-\sqrt{b})\right)
$$

As mentioned earlier, this result does not differ drastically from what is obtained by using $(\bar{\square})$.

On the other hand, it is clear that the use of (7) to estimate the PseudoscalarVector (PV) and Vector-Vector (VV) contributions is dangerous and it could result in an overestimate of these contributions [8]. We can repeat the same procedure carried out with the PP modes. However data is even scarcer in these cases. Measurements for all the PV and VV decays rates as well as a more precise determination of $\Gamma\left(D^{0} \rightarrow K^{+} \pi^{-}\right)$is needed in order to complete the picture of $\Delta m_{D}$ in this approach. However, and as it was already pointed out in [4], it is very likely that the charged pseudoscalar contribution gives a good approximation to the order of magnitude of the effect. This is because, although there are many contributions, their relative signs are not fixed and 
some degree of cancellation is expected. A hint of these cancellations is already present in (11), which includes the interaction between the scale of softening of the effective vertex and the masses. It can be shown that in (11) the contributions of small and large internal masses will tend to have have different signs. This is merely an argument that makes cancellations plausible, but by no means a rigorous proof. It could be argued that several contributions might conspire to give a total long distance contribution to $\Delta m_{D}$ orders of magnitude larger than (13). This scenario cannot be completely excluded in this approach until more data on PV and VV modes is available.

\section{Heavy Quark Effective Theory}

Yet another possible theoretical approach to $D^{0}-\bar{D}^{0}$ mixing is the application of the heavy quark effective theory (HQET). It was first noted in [10] that if one considers the charm quark mass to be much larger than the typical scale of the strong interactions, there would be no nonleptonic transitions to leading order in the resulting effective theory. They would require large momenta to be exchanged between the heavy quark and the light degrees of freedom, a subleading effect in inverse powers of the charm mass. As a consequence there are no new available operators in the low energy theory to produce $\Delta C=2$ transitions. At scales below $m_{c}$ these occur only due to operators present at the matching scale $m_{c}$ plus the action of the renormalization group, which in this picture constitute the only "long distance" effects. Therefore, to leading order in HQET, $\Delta m_{D}$ can be computed from quark operators. The nonperturbative physics enters in the matrix elements of these operators, and in [10, 11] are estimated using naive dimensional analysis. There are three groups of operators in the HQET. The first corresponds to four-quark operators, which are the HQET version of the box diagram. The second group, the six-quark operators, gives a modest enhancement over the first one [10, 11]. Finally, the eight-quark operators give a large enhancement in the matrix elements over the four-quark operators (a factor of $\approx 20$ ), but they are suppressed by an overall factor of $\alpha_{s} / 4 \pi$. This seems to suggest that the large enhancement in $\Delta m_{D}$ over the short distance box diagrams coming from individual contributions and accounted for in the dispersive approach above, is cancelled when all the contributions are summed over in order to make up for the $\alpha_{s} / 4 \pi$ suppression [10]. This is the HQET version of the cancellations among different $S U(3)$ sets (e.g. PP with VV, etc.). The size of the effect is estimated in [11, where QCD corrections and running are properly accounted for. The result is

$$
\frac{\Delta m_{D}}{\Gamma} \approx(1-2) \times 10^{-5}
$$

where the uncertainty comes from the unknown relative signs of the various operators. Thus HQET predicts a value of $\Delta m_{D}$ in the SM that is roughly an order of magnitude smaller than the dispersive estimates (8) and (13). 
Of course the validity of the central HQET assumption, $m_{c} \gg \Lambda_{Q C D}$, can be questioned. After all here $\Lambda_{Q C D}$ is actually a typical hadronic scale, not far below $1 \mathrm{GeV}$. It is not clear what is the size of the corrections. However, the most interesting conclusion is the suggestion that there is a cancellation among the sets of $S U(3)$-related intermediate states. On the other hand, the HQET and the dispersive results are consistent within the experimental errors in the determination of $b$ in DCSD modes.

\section{NEW PHYSICS AND $D^{0}-\bar{D}^{0}$ MIXING}

Proposed high statistics charm experiments are likely to probe $D^{0}-\bar{D}^{0}$ mixing down to $r_{D} \sim 10^{-5}$ [12]. If the long distance contributions in the SM are below this sensitivity, then the question is: are there extensions of the SM that can fill this window and be compatible with all other low energy phenomenology? In this section we review a few examples of new physics scenarios that could produce a signal in these experiments.

\section{Two-Higgs Doublet Models}

As a first example of an extension of the SM we take a Two-Higgs doublet model with natural flavor conservation. That is, there are no tree level FCNC [13]. There will be two neutral scalars, a neutral pseudoscalar and a pair of charged scalars. In what is called Model II in the literature, the latter couples to the fermions as

$$
\mathcal{L}=\frac{g}{\sqrt{2} m_{W}} H^{+}\left\{\cot \beta \overline{\mathcal{U}}_{R} M_{u} V_{\text {ckm }} \mathcal{D}_{L}+\tan \beta \overline{\mathcal{U}}_{L} V_{\text {ckm }} M_{d} \mathcal{D}_{R}+\text { h.c. }\right\}
$$

where $\mathcal{U} \equiv(u, c, t), \mathcal{D} \equiv(d, s, b), M_{u}$ and $M_{d}$ are the diagonal quark mass matrices and $\tan \beta \equiv v_{2} / v_{1}$; with $v_{1}, v_{2}$ the vacuum expectation values of the doublets. Incidentally, this type of couplings to fermions is the same as in the Higgs sector of the Minimal Supersymmetric Standard Model (MSSM), which is addressed separately below. The couplings in (15) induce an additional set of box diagrams where the $W^{ \pm}$is replaced by the charged Higgs. For large values of $\tan \beta$ the $b$ quark is the dominant contribution, giving

$$
\Delta m_{D}^{2 \mathrm{HDM}} \simeq \frac{G_{F}^{2}}{6 \pi^{2}} m_{D} B_{D} f_{D}^{2} \eta_{\mathrm{QCD}}\left|V_{c b} V_{u b}^{*}\right|^{2} m_{b}^{2} \tan ^{2} \beta F\left(\frac{m_{b}^{2}}{m_{H}^{2}}\right)
$$

where $\eta_{\mathrm{QCD}}$ is a $Q C D$ correction and $F(x)$ is a known function of the mass ratios, resulting from the loop integrals. As it is obvious from (16), the effect can be important for large $\tan \beta$. This shows once again how charm meson physics can be complementary with $B$ physics. The radiative decay $b \rightarrow s \gamma$ largely constrains the low $\tan \beta$ region given that, as it can be seen in (15), 


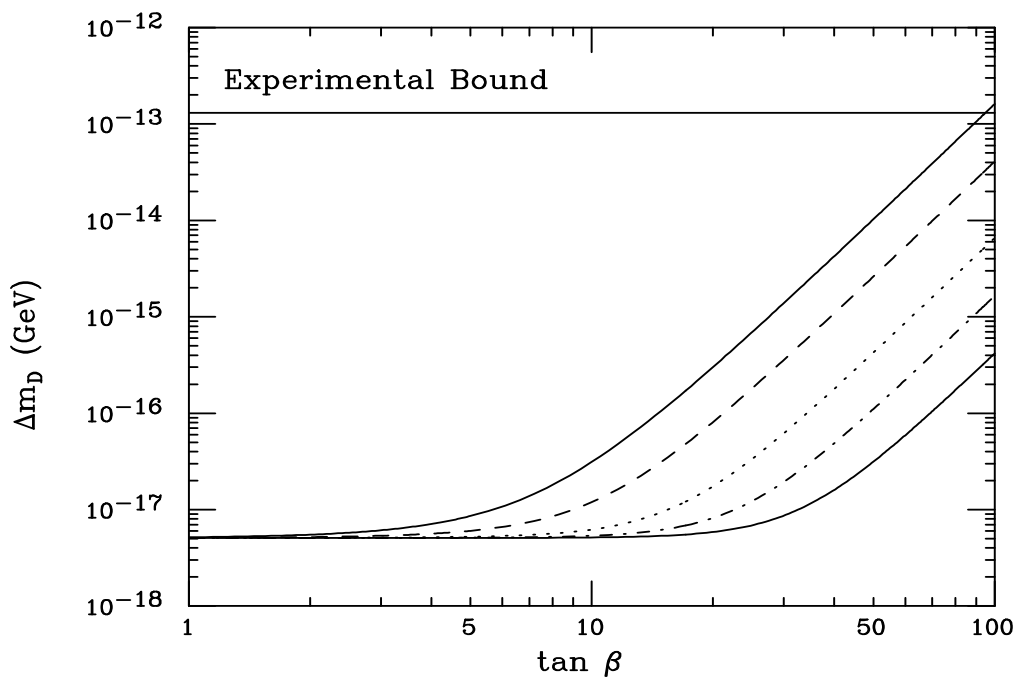

Figure 2: $\Delta m_{D}$ in the two-Higgs doublet model II as a function of $\tan \beta$ and for several charged Higgs masses. From top to bottom $m_{H^{ \pm}}=$ $50,100,250,500,1000 \mathrm{GeV}$.

the top quark mass term amplifies that region of parameter space. As seen in Fig. 2, in the large $\tan \beta$ limit, charged Higgs masses below $m_{H}=250 \mathrm{GeV}$ will give a contribution to which future experiments will be sensitive.

\section{Heavy Down Quark}

The reason why the SM box diagram contributions are so small is a very efficient GIM mechanism: the heaviest quark has very small couplings with $c$ and $u$ and is not so heavy anyway. The obvious question is then: what would happen if there was a fourth down quark in the loop with $Q=-1 / 3$ and a large mass. This could belong to a fourth generation or be just a singlet. Its contribution to the mass difference is

$$
\Delta m_{D}^{b^{\prime}} \simeq \frac{G_{F}^{2} m_{W}^{2}}{6 \pi^{2}} m_{D} B_{D} f_{D}^{2} \eta_{\mathrm{QCD}}\left|V_{c b^{\prime}} V_{u b^{\prime}}^{*}\right|^{2} F\left(\frac{m_{b^{\prime}}^{2}}{m_{W}^{2}}\right)
$$

From direct searches it is known that $m_{b^{\prime}}>85 \mathrm{GeV}$ [5]. In the case of $b^{\prime}$ belonging to a fourth generation, the couplings to the second and first generations are constrained by the possible "leakage" of the CKM matrix from unitarity. The mixing factors must satisfy

$$
\left|V_{u b^{\prime}}\right|<0.08, \quad\left|V_{c b^{\prime}}\right|<0.6
$$

although smaller mixing factors are expected. The effect of the $b^{\prime}$ quark is shown in Fig. 3. If the mixing factors are not too small a heavy $b^{\prime}$ could give a large effect, saturating the current experimental limit. 


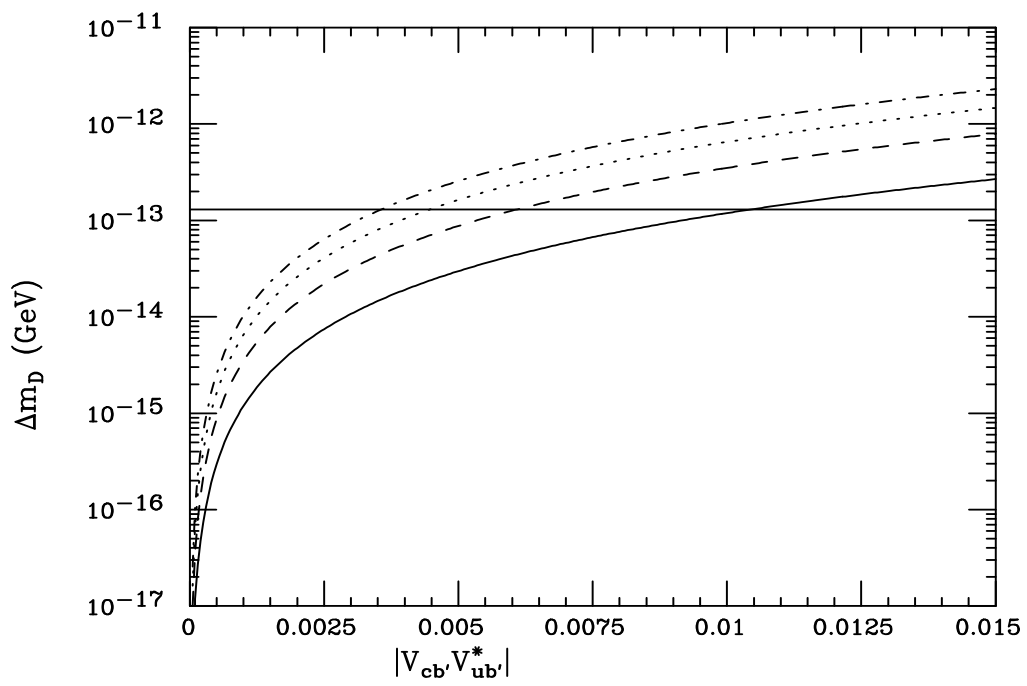

Figure 3: The contributions to $\Delta m_{D}$ of a heavy $Q=-1 / 3$ quark, as a function of the mixing factor in a fourth-generation model. From top to bottom $m_{b^{\prime}}=$ 400,300, 200, $100 \mathrm{GeV}$. The horizontal line shows the current experimental limit.

\section{Tree Level FCNC}

Tree-level FCNC are severely constrained by $K^{0}-\bar{K}^{0}$ and $B^{0}-\bar{B}^{0}$ mixings and decays like $K^{+} \rightarrow \pi^{+} \nu \bar{\nu}$ and $B \rightarrow X \ell^{+} \ell^{-}$[5]. However, it is possible to imagine scenarios where the up-quark sector is treated differently. Some of these new physics scenarios predict large $D^{0}-\bar{D}^{0}$ mixing effects.

A first example is to relax the requirement of flavor conservation in multiHiggs models. There will be tree-level flavor changing couplings of the neutral Higgses [14]. The form of the flavor conserving couplings to fermions suggests the parametrization of the flavor changing couplings given by

$$
C_{i j} \sim \frac{\sqrt{m_{i} m_{j}}}{v_{\mathrm{w}}} \delta_{i j}
$$

where $i, j$ are the quark labels (e.g. $u, c, t$ ); and $v_{\mathrm{w}} \simeq 246 \mathrm{GeV}$. The $c-u$ coupling would then induce a contribution to the mass difference of the form

$$
\Delta m_{D}^{h^{0}}=\frac{5}{12} f_{D}^{2} B_{D} m_{D} \frac{m_{c} m_{u}}{v_{\mathrm{w}}^{2}} \frac{\delta_{c u}^{2}}{m_{h}^{2}}
$$

where $m_{u}$ is the current quark mass. The experimental limit is saturated for reasonable values of $\delta_{c u} \sim(0.2-1)$ and $m_{h} \sim(50-250) \mathrm{GeV}$.

Another interesting example is that of theories of dynamical symmetry breaking with large GIM violations, such as TopColor models [15]. Here the 
top quark gets a large mass after the breaking down to $Q C D$ of a group strongly coupled to the third generation. This generates a set of pseudoGoldstone bosons (top-pions) that couple to the up-quark sector. After the quark weak eigenstates are rotated to the mass eigenstates, there will be flavor changing neutral couplings mediated by the top-pions of the form

$$
C_{i j} \sim \frac{m_{t}}{\sqrt{2} f_{\tilde{\pi}}} U_{L}^{t i} U_{R}^{t j}
$$

where $f_{\tilde{\pi}} \simeq 50 \mathrm{GeV}$ is the top-pion decay constant and $U_{L}$ and $U_{R}$ are the mass matrices of the left and right up-quark sector respectively. These couplings lead to a mass difference given by

$$
\Delta m_{D}^{\text {t.c. }} \simeq \frac{5}{12} f_{D}^{2} B_{D} m_{D} \frac{m_{t}^{2}}{2 f_{\tilde{\pi}}^{2}} \frac{U_{L}^{t c} U_{R}^{t u} U_{L}^{t u} U_{R}^{t c}}{m_{\tilde{\pi}}^{2}}
$$

The mass matrices are not determined in general by the model but must obey, as in the SM, $V_{\text {ckm }}=U_{L}^{\dagger} D_{R}$. This suggests that a possible anzatz for $U_{L, R}$ is to take the "squared root" of $V_{\text {ckm }}$. This prescription gives, for instance, $U_{L}^{t c} \simeq(1 / 2) V_{c b}$. Following this in (22) and taking $m_{\tilde{\pi}} \simeq 200 \mathrm{GeV}$ [15], gives $\Delta m_{D}^{\text {t.c. }} \simeq 8 \times 10^{-14} \mathrm{GeV}$, right below the current experimental limit. In principle, other textures can be chosen that would not give such a large effect.

\section{Supersymmetry}

In addition to the box diagrams involving charged Higgses, there will be contributions from squarks + (gluinos, charginos or neutralinos) box diagrams. These vanish if the down squarks are degenerate. This is just a statement derived from GIM cancellations. However, there could be flavor changing, radiatively generated mass insertions [16]. They are thought to be small in the MSSM, but could be large in non minimal models [17]. For instance, the action of these mass insertions would allow for squark-gluino box diagrams. The resulting $\Delta C=2$ hamiltonian is

$$
\begin{aligned}
\mathcal{H}_{\Delta C=2}^{\mathrm{SUSY}}=\frac{\alpha_{s}^{2}}{216 \tilde{m}_{0}} & \left\{\frac{\delta \tilde{m}_{u_{L} c_{L}}^{2}}{\tilde{m}_{0}^{2}} G\left(\frac{m_{\tilde{g}^{2}}}{\tilde{m}_{0}^{2}}\right)\left(\bar{u}_{L} \gamma_{\mu} c_{L}\right)\left(\bar{u}_{L} \gamma^{\mu} c_{L}\right)\right. \\
+ & (R R)^{2}+(L L)(R R)+(L R)^{2}+(L R(L R)\}
\end{aligned}
$$

where $\tilde{m}_{0}$ is the universal scalar mass, $m_{\tilde{g}}$ is the gluino mass, $\delta \tilde{m}_{u_{L} c_{L}}$ characterizes the mass insertion and the $G(x)$ 's are known functions. This way, the experimental upper limit on $\Delta m_{D}$ can be translated into limits for the various terms entering in (23). These are

$$
\frac{\delta \tilde{m}_{u_{A} c_{B}}^{2}}{\tilde{m}_{0}^{2}}<\left\{\begin{array}{cl}
(0.2)^{2} & , \text { for }(L L)^{2},(R R)^{2} \\
\left(3.6 \times 10^{-2}\right)^{2} & , \text { for }(L L)(R R) \\
\left(5 \times 10^{-2}\right)^{2} & , \text { for }(L R)^{2},(R L)^{2} \\
(0.1)^{2} & , \text { for }(L R)(L R)
\end{array}\right.
$$


Thus, the current experimental limit is already sensitive to non minimal SUSY effects.

There are several other extensions of the SM that would saturate the current experimental limit or at least give a signal in high statistics charm experiments. The above list is by no means exhaustive and is mainly intended to illustrate how the effect comes about in a variety of theories.

\section{CP VIOLATION}

The $D$ system is not particularly sensitive to $\mathrm{CP}$ violation in the SM to the extent the $K$ and $B$ mesons are. Once again, this could imply there is a window of observation of new physics effects. In what follows we discuss some of the general features of $\mathrm{CP}$ violation in $D$ mesons rather than going into specific calculations, both in the SM and beyond.

\section{Direct CP Violation}

The occurrence of direct $\mathrm{CP}$ violation requires the concurrence of both weak and strong relative phases between two or more amplitudes contributing to a given final state. In the SM, relative weak phases can only be obtained in Cabibbo suppressed decays, for instance, via the interference between spectator and penguin amplitudes. To estimate the size of the $\mathrm{CP}$ asymmetries this would generate, we write

$$
\begin{aligned}
& a_{\mathrm{CP}} \sim \frac{\operatorname{Im}\left[V_{c d} V_{u d}^{*} V_{c s} V_{u s}^{*}\right]}{\lambda^{2}} \sin \delta_{\mathrm{st}} \frac{P}{S} \\
& \sim A^{2} \eta \lambda^{4} \sin \delta_{\mathrm{st}} \frac{P}{S} \quad \leq 10^{-3}
\end{aligned}
$$

where $\delta_{\text {st }}$ is the strong relative phase between the penguin and the spectator amplitudes, and $A \sim 1$ and $\eta$ are CKM parameters in Wolfenstein's parametrization. Specific model calculations for $D \rightarrow K K, \pi \pi, K^{*} K$, threebody modes, etc. yield this order of magnitude for the effect. New physics

could enter, for instance, through large phases in the penguin diagram. This could give very large asymmetries of the order of one percent or larger. On the other hand, an even cleaner window are the Cabibbo allowed decays. These modes do not have two amplitudes with different weak phases and therefore the CP asymmetry is zero in the SM. There are new physics scenarios that provide extra phases and could give asymmetries as large as one percent. This is for instance the case in some left-right symmetric models [18]. The current experimental sensitivities for various modes is in the vicinity if $10 \%$ [19. 


\section{Indirect CP Violation}

The interaction between mixing and $\mathrm{CP}$ violation in $D$ mesons has recently received a lot of attention in the literature [20, 6, 0]. Here we shall focus only on one aspect, which can be condensed in the following question: if mixing is large (e.g. right below the current experimental limit) should $\mathrm{CP}$ violation in $D$ decays be large ? The question is motivated by the fact that in $B$ decays the large $B^{0}-\bar{B}^{0}$ mixing is known to give large $\mathrm{CP}$ asymmetries. We first define the time-evolved states in the usual way, as

$$
\begin{aligned}
\left|D^{0}(t)\right\rangle & =g_{+}(t)\left|D^{0}\right\rangle+\frac{q}{p} g_{-}(t)\left|\bar{D}^{0}\right\rangle \\
\left|\bar{D}^{0}(t)\right\rangle & =\frac{p}{q} g_{-}(t)\left|D^{0}\right\rangle+g_{+}(t)\left|\bar{D}^{0}\right\rangle
\end{aligned}
$$

with

$$
\frac{p}{q} \equiv \sqrt{\frac{M_{12}-i \Gamma_{12}}{M_{12}^{*}-i \Gamma_{12}^{*}}}
$$

and the time evolution given by

$$
g_{ \pm}(t)=\frac{1}{2} e^{\left(-\frac{\Gamma_{L}}{2} t+i m_{L} t\right)}\left[1 \pm e^{\left(-\frac{\Delta \Gamma_{D} t}{2}+i \Delta m_{D} t\right)}\right]
$$

We also need to define the amplitudes

$$
\begin{aligned}
& A \equiv\left\langle f\left|H_{w}\right| D^{0}\right\rangle \quad ; \quad B \equiv\left\langle f\left|H_{w}\right| \bar{D}^{0}\right\rangle \\
& \bar{A} \equiv\left\langle\bar{f}\left|H_{w}\right| \bar{D}^{0}\right\rangle \quad ; \quad \bar{B} \equiv\left\langle\bar{f}\left|H_{w}\right| D^{0}\right\rangle
\end{aligned}
$$

and the ratio

$$
\bar{\rho} \equiv \frac{A}{B}
$$

To simplify notation we consider the case when $f=C P$ eigenstate (e.g. $\pi \pi, K K$, etc). If mixing is a large effect, let us say right below the current upper limit, and therefore due to new physics contributions then it is very likely that $\Delta m_{D} / \Gamma \gg \Delta \Gamma_{D} / \Gamma$ is a very good approximation: non standard contributions to $\Delta \Gamma_{D}$ are constrained by actual branching ratios. Under these assumptions the asymmetry takes the form

$$
a_{\mathrm{CP}} \simeq \frac{1-\left|\frac{q}{p} \bar{\rho}\right|^{2}-2 \frac{\Delta m_{D}}{\Gamma} \operatorname{Im}\left[\frac{q}{p} \bar{\rho}\right]}{\left(1+\left|\frac{q}{p} \bar{\rho}\right|^{2}\right)\left(1+\left(\frac{\Delta m_{D}}{\Gamma}\right)^{2}\right)}
$$

Small direct CP violation implies $|(q / p) \bar{\rho}|^{2} \simeq 1$. This leads to

$$
a_{\mathrm{CP}} \simeq \frac{\Delta m_{D}}{\Gamma} \times \operatorname{Im}\left[\frac{q}{p} \bar{\rho}\right] \simeq \frac{\Delta m_{D}}{\Gamma} 2 \eta A
$$


where the last step follows in the SM and from considering the

contribution of the $b$ quark to the imaginary part of the box diagram.

The resulting asymmetry can be then of the order of $\Delta m_{D} / \Gamma$ even if only

the SM phases intervene. However in models giving large mass

differences it is also likely that there will be additional

$\mathrm{CP}$ violating phases.

These could be present in the non SM contributions to the mass difference and they mostly affect $q / p$ and not $\bar{\rho}$. Several non standard scenarios for generating these additional phases are discussed in [20].

\section{RARE AND RADIATIVE DECAYS}

Let us first address the distinction made between rare and radiative decays. Radiative weak decays of charm mesons do no effectively test the SM. To see this let us take the transitions governed by the short distance flavor changing vertex $c \rightarrow u \gamma$. In the SM they occur only at one loop through the electromagnetic penguin, analogous to $b \rightarrow s \gamma$. However in this case, the inclusive branching ratio, even after very large $Q C D$ corrections, is very small: $B(c \rightarrow u \gamma) \simeq 10^{-12}$ [21]. This, however, does not constitute a window for new physics. There are more mundane contributions to the corresponding exclusive processes, like $D^{0} \rightarrow \rho^{0} \gamma$, that do not arise from short distance physics. These "long" distance contributions can be thought of as coming from either pole diagrams (arising from quark exchange) and vector meson dominance diagrams, all of which are not calculable from first principles but can be estimated in models to give $B\left(D^{0} \rightarrow \rho^{0} \gamma\right) \simeq(1-5) \times 10^{-6}$ [21]. This large rates preclude the use of these modes as SM tests. On the other hand, a better theoretical understanding of these modes is interesting in its own right as well as in order to understand possible long distance contamination in radiative $B$ decays [22]. The availability of several decay modes $-D^{0} \rightarrow \rho^{0} \gamma, D^{0} \rightarrow \bar{K}^{*} \gamma, D_{s} \rightarrow \rho^{+} \gamma$, etc.- at branching fractions of $\mathcal{O}\left(10^{-6}\right)$ or larger will improve our knowledge of strong dynamics at the charm scale [23].

Truly rare decays are those whose SM rates are extremely small or simply zero. Most of them proceed through FCNC induced at one loop in the SM. The simplest example is $D^{0} \rightarrow \ell^{+} \ell^{-}$, with $\ell=e$ or $\mu$. Their branching ratios are smaller than $10^{-15}$, even after long distance contributions are taken into account. There are experimentally clean and any signal in any of these channels would imply new physics. However the helicity suppression is a factor of $\sim 10^{-3}$ in the case of $\mu$, and $10^{-7}$ for the $e$. Modes without this suppression, like $D \rightarrow X \ell^{+} \ell^{-}$and $D \rightarrow X \nu \bar{\nu}$, are more likely to show the first signals if, for instance, there is a new physics mechanism underlying the short distance transition $c \rightarrow u$. In the SM their branching ratios are expected to be of 
$\mathcal{O}\left(10^{-8}\right)$ or smaller in the charged lepton cases and negligibly small in the neutrino modes. New upper limits in the $D^{+} \rightarrow \pi^{+} \ell^{+} \ell^{-}$channels have been recently reported by E791 [24]: $B_{\mu \mu}<1.8 \times 10^{-5}$ and $B_{e e}<6.6 \times 10^{-5}$. These are already constraining extensions of the SM in a way complementary with mixing. Predictions for new physics scenarios can be found in several places in the literature [25], but it is necessary to update them and most importantly to take into account other pieces of phenomenology now available.

\section{CONCLUSIONS}

We have seen that charm meson physics offers several opportunities to observe the effects of new physics. This is mainly due to the suppression of the signals in the SM. In the case of $D^{0}-\bar{D}^{0}$ mixing, the estimate of the long distance SM contribution is very uncertain. However, the question of interest at the moment is whether

$$
r_{D}^{\mathrm{SM}}<10^{-5}
$$

is a correct upper limit. This is relevant because is the planned sensitivity of future high statistics charm experiments [12]. If $r_{D}^{S M}$ is $10^{-10}$ or $10^{-7}$ is an issue theorists should worry about, but it will not affect the interpretation of the outcome of these experiments. The validity of (31) is not a settled question among theorists. We have seen two approaches to $r_{D}^{\mathrm{SM}}$ satisfying (31): a dispersive approach and the HQET approach. However, these are approximate calculations and there are those who point out that (6) with a unit coefficient is another way of estimating the effect [26]. The high end of this estimate violates (31). The theoretical community should make an effort to resolve this outstanding problem. More data in nonleptonic $D$ decays is needed in order to see if there is a pattern of cancellations as suggested by the HQET. In the meantime, experiments might provide with an independent way

of deciding on the origin of an observation of $r_{D}$ : the direct measurement of the lifetime difference $\Delta \Gamma_{D}$. This can be done, assuming CP conservation, by looking at the decay time distribution of $D$ decays to $\mathrm{CP}$ even and odd final states [27]. The difference of the slopes is proportional to $\Delta \Gamma_{D}$. This quantity is not prone to get contributions from new physics but rather to be entirely given by the SM: after all it is a sum over real intermediate states. Moreover, it should be of the same order of magnitude as the long distance contributions to $\Delta m_{D}$. Thus, not only would this allow the separation of $\Delta m_{D}$ and $\Delta \Gamma_{D}$ but also, for instance, point at new physics if $\Delta m_{D}$ is observed and $\Delta \Gamma_{D}$ is not seen at the corresponding level. Considering how many extensions of the $\mathrm{SM}$ saturate the experimental limit, this program makes $D^{0}-\bar{D}^{0}$ mixing an important window for new physics in the future. 
With respect to $\mathrm{CP}$ violation, we have seen that asymmetries at the level of one percent would signal new physics. We also pointed out that $\mathrm{CP}$ violation due to mixing will be enhanced by new physics only if both $\Delta m_{D}$ and

the entering phases, both from the SM and/or new physics, are large.

For rare decays a lot more work is needed in order to establish what level of branching ratios are allowed in each new physics scenario once all the constraints from low energy phenomenology are factored in.

\section{AKNOWLEDGEMENTS}

This presentation reflects work in collaboration with Eugene Golowich, JoAnne Hewett and Sandip Pakvasa. I would also like to thank Jose Repond and the rest of the organizers of the workshop for their excellent effort. This work was supported by the U.S. Department of Energy.

\section{References}

[1] J. C. Anjos et al., Phys. Rev. Lett. 60, 1239 (1988).

[2] A. Datta and D. Kumbhakar, Z. Phys. C27, 515 (1985).

[3] J. F. Donoghue, E. Golowich and B. R. Holstein, Phys. Lett. B135, 481 (1984).

[4] J. F. Donoghue, E. Golowich, B. R. Holstein and J. Trampetić, Phys. Rev. D33, 179 (1986).

[5] L. Montanet et al., Phys. Rev. D50, 1173 (1994).

[6] L. Wolfenstein, Carnegie Mellon Univ. preprint CMU-HEP-95-04 (1995), hep-ph/9505285.

[7] T. E. Browder and S. Pakvasa, Univ. of Hawaii preprint UH 511-828-95 (1995).

[8] T. A. Kaeding, LBL preprint LBL-37224 (1995).

[9] G. Burdman, E. Golowich, J. Hewett and S. Pakvasa, in preparetion.

[10] H. Georgi, Phys. Lett. B297, 353 (1992).

[11] T. Ohl, G. Ricciardi and E. H. Simmons, Nucl. Phys. B403, 605 (1993). 
[12] D. M. Kaplan, in these Proceedings. See also R. J. Morrison, in Proceedings of the Charm 2000 Workshop, Ed. D. M. Kaplan and S. Kwan, FERMILAB-Conf-94/190, (1994).

[13] S. L. Glashow and S. Weinberg, Phys. Rev. D15, 1958 (1977).

[14] S. Pakvasa and H. Sugawara, Phys. Lett B73, 61 (1978);

L. Hall and S. Weinberg, Phys. Rev. D48, 979 (1993).

[15] C. T. Hill, Phys. Lett. B345, 483 (1995).

[16] J. S. Hagelin, S. Kelley, T. Tanaka, Nucl. Phys. B415, 293 (1994); Mod. Phys. Lett. A8,2737 (1993).

[17] Y. Nir and N. Seiberg, Phys. Lett. B309, 337 (1993).

[18] M. Gronau and S. Wakaizumi, Phys. Rev. Lett. 68, 1814 (1992);

A. Le Yaouanc, L. Oliver and J.-C. Raynal, Phys. Lett. B292, 353 (1992).

[19] P. L Frabetti et al., Phys. Rev. D50, 2953 (1994);

J. Bartelt et al., CLEO preprint CLNS 95/1333 (1995).

[20] G. Blaylock, A. Seiden and Y. Nir, Univ. of Sta. Cruz preprint SCIPP 95/16 (1995).

[21] G. Burdman, E. Golowich, J. Hewett and S. Pakvasa, FERMILAB-Pub94/412-T (1995).

[22] E. Golowich and S. Pakvasa, Phys. Rev. D51,1215 (1995).

[23] I. I. Bigi, these Proceedings.

[24] E. M. Aitala et al., Fermilab preprint FERMILAB-Pub-95/142-E (1995).

[25] For the effect of lepto-quarks see, W. Buchmuller and D. Wyler, Phys. Lett. B177, 377 (1986) and more recently M. Leurer, Phys. Rev. Lett. 71, 1324 (1993).

[26] L. Wolfenstein, Phys. Lett. B164, 170 (1985).

[27] T. Liu, these proceedings and in Proceedings of the Charm 2000 Workshop, Ed. D. M. Kaplan and S. Kwan, FERMILAB-Conf-94/190, (1994). 